\title{
Photometric observations of distant active comets ${ }^{\star}$
}

\author{
Gy. M. Szabó ${ }^{1}$, B. Csák ${ }^{1,2}$, K. Sárneczky ${ }^{3}$, and L. L. Kiss ${ }^{1}$ \\ 1 Department of Experimental Physics \& Astronomical Observatory, University of Szeged, 6720 Szeged, \\ Dóm tér 9, Hungary \\ 2 Department of Optics \& Quantum Electronics \& Astronomical Observatory, University of Szeged, POB 406, \\ 6701 Szeged, Hungary \\ 3 Department of Physical Geography, ELTE University, 1088 Budapest, Ludovika tér 2, Hungary \\ Received 21 September 2000 / Accepted 17 April 2001
}

\begin{abstract}
We present CCD $V R_{\mathrm{C}}$ observations of 6 distant comets located at heliocentric distances of 3.4-7.2 AU. Time-series data were obtained on three nights in July, 2000 covering 16 hours. Each comet was observed after the perihelion, when a lower activity was expected. Contrary to expectation, we found well-defined circular comae and extended tails visible out to a few (3-5) arcmin. We detected a quasi-cyclic light variation of C/1999 J2, while $\mathrm{C} / 1999 \mathrm{~N} 4$ showed some hints of a more complex variation. C/2000 K1 was constant to \pm 0.04 mag during the observing run. The standard $V$ and $R_{\mathrm{C}}$ data were used to estimate nuclear diameters, while the colour indices implied a slighly reddish $\left(V-R=0{ }^{\mathrm{m}} 68, \mathrm{C} / 2000 \mathrm{~K} 1\right)$, a neutral $(V-R=0 . \mathrm{m} 47, \mathrm{C} / 1999 \mathrm{~N} 4)$ and a slightly bluish $\left(V-R=0{ }^{\mathrm{m}} 25, \mathrm{C} / 1999 \mathrm{~J} 2\right)$ coma. Simple fits of the surface brightness distributions are also presented enabling order of magnitude estimates of nuclear radii. Beside the time-series observations, further single-shot observations of three faint comets are briefly described.
\end{abstract}

Key words. solar system - comets: individual: C/1999 J2, C/1999 N4, C/2000 K1

\section{Introduction}

The overwhelming majority of cometary studies are based on ground-based or space observations of bright comets around or near to their perihelion. Direct imaging reveals the inner structure of the coma, which usually hides the nucleus itself. There has been a small number of papers dealing with distant ( $R \geq 5 \mathrm{AU}$ ) comets (e.g. O'Ceallaigh et al. 1995; Lowry et al. 1999), and consequently, there is a serious lack of information on the behaviour of these objects. On the other hand, time-series observations may give constraints on the period of rotation and related effects (Jewitt 1992).

Optical photometry of sunlight scattered from the nucleus was first attempted by Fay \& Wisniewsky (1978) on comet $6 \mathrm{P} / \mathrm{d}$ 'Arrest. The approach is identical to that used in the asteroid studies. Further CCD photometry using very small aperture radii has been used to study the rotation of some comets by Jewitt \& Meech (1985), Jewitt (1990), Meech et al. (1993, 1997), Licandro et al. (2000a, 2000b). Licandro et al. (2000a) discussed in detail rotation and shape models based on the lightcurves. Additional re-

Send offprint requests to: L. L. Kiss,

e-mail: 1.kiss@physx.u-szeged.hu

* Based on observations taken at the German-Spanish Astronomical Centre, Calar Alto, operated by the Max-PlanckInstitute for Astronomy, Heidelberg, jointly with the Spanish National Commission for Astronomy. duction has to be done with respect to the seeing effects as the variation in seeing may cause a virtual light variation of the inner coma with an amplitude of some tenths of a magnitude.

The nucleus is usually well embedded in the coma and two basic approaches exist to determine the nuclear diameter. The most efficient one is the use of high spatial resolution observations of closely passing comets with the HST (e.g. Lamy \& Tóth 1995; Lamy et al. 1998, 1999). The other commonly used method is observing distant nuclei in quiescence, when the coma is effectively absent (Luu \& Jewitt 1992; O'Ceallaigh et al. 1995; Boehnhardt et al. 1999). Very recently, the nuclear thermal radiation of some comets was studied in the infrared with help of ISOCAM observations, resulting in independent diameter estimates (Jorda et al. 2000).

The new observing strategies by automatic telescopes (e.g. project LINEAR) and dedicated large instruments (Hainaut \& Meech 1997) led to regular discoveries of relatively bright, large perihelion-distance comets, often more months or a year before the perihelion passage. Thus, one can study cometary activity in such regions that were unavailable even a decade ago. The main aim of our work is to contribute to this topic with new CCD observations of three comets located between 5.5-7.2 AU - C/1999 J2 (Skiff), C/1999 N4 (LINEAR) and C/2000 K1 (LINEAR). Beside the absolute $V R_{\mathrm{C}}$ photometry we took timeseries observations in order to detect rotational effects. 
Table 1. The journal of observations. ( $R$ - heliocentric distance; $\Delta$ - geocentric distance; $E$ - elongation; $\lambda$ - ecliptic longitude; $\beta$ - ecliptic latitude; $\alpha$ - solar phase angle; aspect data are referred to $2000.0 ; \mu_{R}-\mathrm{mag} / \operatorname{arcsec}^{2}$ in $R$-band).

\begin{tabular}{|c|c|c|c|c|c|c|c|c|c|c|c|}
\hline Date & $\mathrm{RA}$ & Dec & $R(\mathrm{AU})$ & $\Delta(\mathrm{AU})$ & $E$ & $\lambda\left({ }^{\circ}\right)$ & $\beta\left(^{\circ}\right)$ & $\alpha\left(^{\circ}\right)$ & $\operatorname{see}\left({ }^{\prime \prime}\right)$ & $\operatorname{sky}\left(\mu_{R}\right)$ & $\exp (\mathrm{s})$ \\
\hline \multicolumn{12}{|l|}{ C/1999 F2 } \\
\hline 2000 June 30 & 1705 & +3045 & 5.512 & 4.728 & 137.6 & 205.3 & 63.7 & 7.3 & 2.1 & 20.2 & 240 \\
\hline \multicolumn{12}{|l|}{ C/1999 J2 } \\
\hline 2000 July 06 & 1520 & +3229 & 7.135 & 6.836 & 104.2 & 326.2 & 48.1 & 8.0 & 1.5 & 19.8 & 240 \\
\hline 2000 July 09 & 1519 & +3203 & 7.136 & 6.868 & 101.9 & 326.1 & 47.7 & 8.0 & 2.1 & 19.5 & 240 \\
\hline \multicolumn{12}{|l|}{$\mathrm{C} / 1999 \mathrm{~N} 4$} \\
\hline 2000 July 04 & 1623 & +0535 & 5.514 & 4.771 & 133.9 & 297.2 & 26.7 & 8.0 & 1.6 & 20.7 & 240 \\
\hline 2000 July 06 & 1621 & +0532 & 5.515 & 4.794 & 132.0 & 297.2 & 26.7 & 8.0 & 1.4 & 19.8 & 240 \\
\hline \multicolumn{12}{|l|}{ C/1999 Т2 } \\
\hline 2000 July 02 & 2129 & +6025 & 3.351 & 3.318 & 90.5 & 249.6 & 53.3 & 17.7 & 1.9 & 20.2 & 90 \\
\hline \multicolumn{12}{|l|}{$\mathrm{C} / 2000 \mathrm{H} 1$} \\
\hline 2000 June 30 & 1527 & +4818 & 3.890 & 3.643 & 96.4 & 347.4 & 66.7 & 15.1 & 2.1 & 20.2 & 240 \\
\hline \multicolumn{12}{|l|}{ C/2000 K1 } \\
\hline 2000 July 04 & 1604 & +1305 & 6.429 & 5.798 & 125.4 & 304.4 & 33.2 & 7.5 & 1.6 & 20.7 & 180 \\
\hline
\end{tabular}

The paper is organised as follows. The observations are described in Sect. 2, while Sect. 3 deals with the analysis and detailed observational results. A brief discussion is given in Sect. 4.

\section{Observations}

Johnson $V$ and $R_{\mathrm{C}}$ filtered CCD observations were carried out at Calar Alto Observatory (Spain) on three nights in July, 2000 (4th, 6th and 9th). The instrument used was the 1.23-m telescope equipped with the SITe\#2b CCD camera (2048x2048 pixels giving an angular resolution of $0 . ! 49 /$ pixel). The projected sky area is $16 ! 0 \times 16 ! 0$, 10 . $0 \times 10$ ' 0 unvignetted.

The targets were selected following a few practical restrictions. Since the main aim was to observe distant comets with small expected coma contributions, we chose every visible comet at solar distances larger than 5 AU. The central brightness was restricted to be brighter than $19.0 \mathrm{mag}$, since time-series observations were planned to reveal rotation. Four comets remained as possible targets, and two further comets at a solar distance between 3 and $4 \mathrm{AU}$ were added as auxiliary candidates. Finaly, six comets were examined.

We captured all of them a few days before starting the comet observations (on June 30th and July 2nd) in order to check which comets would be well-suited for our purposes. Three of them turned out to be unsuitable for the detailed analysis, thus we only characterize their comae and tails. Three objects remained as final targets for the morphological and photometric studies.

The exposure time was limited by two factors: firstly, the comets were not allowed to move more than two times the $F W H M$ of the stellar profiles (varying from night to night) and secondly, the signal-to-noise $(S / N)$ ratio had to be at least 20 . This latter parameter was estimated comparing the peak pixel values with the sky background during the observations. The adapted exposure time was $240 \mathrm{~s}$. The observing log is summarized in
Table 1, listing also the main geometric parameters and sky conditions.

The CCD frames were reduced with standard tasks in IRAF. We obtained flat-fields taken during the evening twillight, and a master flat-field was formed with the task flatcombine. The photometric reductions were done with the corresponding tasks in IRAF/APPHOT. The trailed images and the presence of faint comae did not permit the use of psf-photometry. Therefore, a simple aperture photometry was performed. The applied differential photometry consisted of using two stars nearby as comparison and check stars. The cometary magnitudes are relative to the ensemble mean of the comparison, thus improving the precision of the differential data. We have carefully examined the aperture choice and a 2.' 6 diameter was accepted with respect to the mean $F W H M$ and its doubled value. The time-series accuracy was estimated by selecting two nearby stars at similar brightnesses to those of the nuclei for the comparison and check stars. The usual scatter of comp minus check measurements was about \pm 0 . 02 .

Since all three nights were photometric, we could make an absolute "all-sky" photometry using photometric standard stars taken from Landolt (1992). The standard field of PG1633+099 used was fairly close to the celestial positions of the observed comets. This field contains five standard stars covering $V$ magnitudes between 12 m969 and $15{ }^{\mathrm{m}} 256$ and $V-R$ colour between $-0{ }^{\mathrm{m}} 093$ and 0.618 . The extinction was monitored by observing an A-type standard at different air-masses, and the nightly zeropoints of the standard transformations were determined with the other standards. The standard deviation of the linear fits is $\pm 0.02 \mathrm{mag}$, implying similar precision for the absolute values.

Another important correction specific for comet photometry was also applied. Licandro et al. (2000a) discussed the effects of the varying seeing on the photometry of blurred diffuse surfaces, such as those observed in comets. These authors outlined the following method: the actual seeing is determined in every CCD frame by 


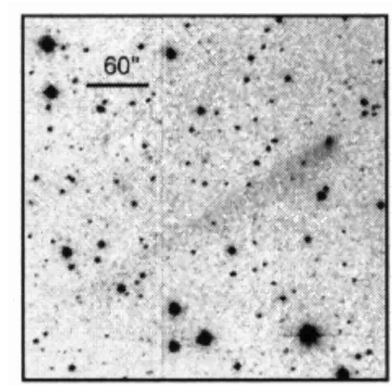

C/1999 F2

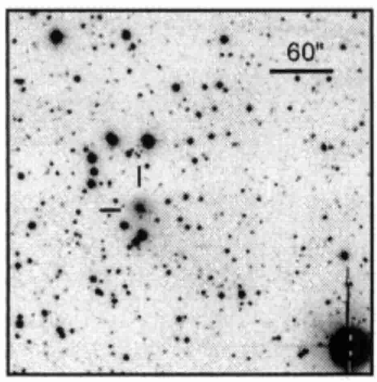

C/1999 T2

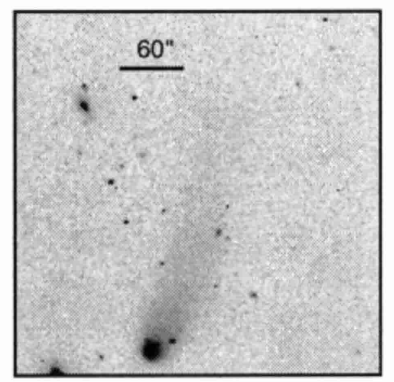

$\mathrm{C} / 1999 \mathrm{~J} 2$

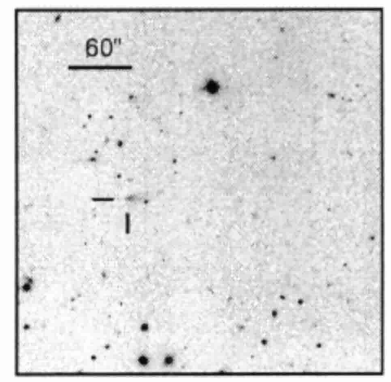

$\mathrm{C} / 2000 \mathrm{H} 1$

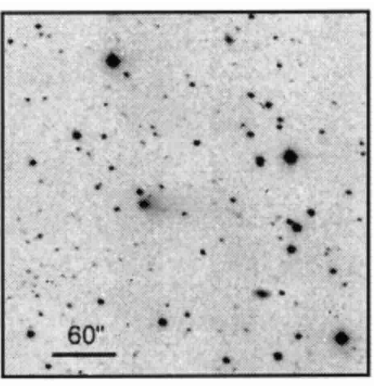

C/1999 N4

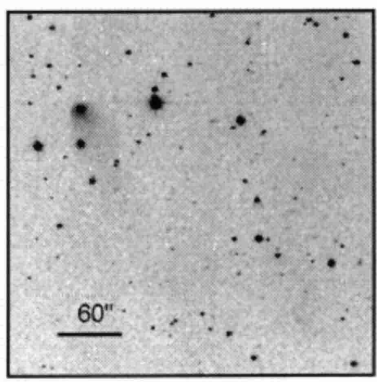

$\mathrm{C} / 2000 \mathrm{~K} 1$

Fig. 1. $R$-filtered images of the observed comets (C/1999 F2: June 30, C/1999 J2: July 9, C/1999 N4: July 4, C/1999 T2: July 2, C/2000 H1: June 30, C/2000 K1: July 4). North is up, west is left. The images were scaled nonlinearly to enhance the visibility of diffuse outer regions.

examining stellar profiles. Then a seeing-magnitude relation is found with help of artificially blurred images of a non-variable comet. In this way the "seeing-effect" on the magnitude determination carried out in the inner coma can be corrected for each observed frame with varying seeing. This "seeing-subtraction" removes the some part of the atmospheric effect and the remaining variation can be attributed to the comet itself. The typical corrections did not exceed $0 .{ }^{\mathrm{m}} 1$. The procedure adds a further $\sim 0^{\mathrm{m}} 01$ noise to the data and including all of the mentioned uncertainties, the photometric accuracy is estimated to be \pm 0.05 . The data reduction ends with the correction for the light time $^{1}$.

\section{Analysis and results}

In this section we discuss the applied analysis methods and individual results for the observed comets. After describing the morphology we discuss their magnitudes and make an attempt to estimate the nuclear radii. We also analyze the time series data.

As can be seen in Fig. 1, despite the large solar distances, the observed comets are fairly active. The general appearance is dominated by a small circular coma and a faint, considerably long tail. Although this behaviour is not typical for Short Period Comets, the Long

\footnotetext{
${ }^{1}$ Original data are available in electronic form at CDS via anonymous ftp to

cdsarc.u-strasbg.fr $(130.79 .128 .5)$ or via

http://cdsweb.u-strasbg.fr/cgi-bin/qcat?J/A+A/374/712
}

Period Comets display exactly this appearance, keeping the level of activity over a very wide range of distances (Meech 1991). The presented images, that are single exposures, were enhanced nonlinearly to emphasize the faint tail extensions.

To derive the physical length of tails one has to estimate their apparent size. The end of the tail was defined where the signal-to-noise ratio of the surface brightness was 2 on the composite images (4 individual exposures coadded). The apparent tails are visible out to $5 ! 8(\mathrm{C} / 1999$ F2), 5!2 (C/1999 J2), 3'0 (C/1999 N4), 0!8 (C/1999 T2), $0 ! 3(\mathrm{C} / 2000 \mathrm{H} 1)$ and 5!9 (C/2000 K1).

The measured position angles (PA) are the following (antisolar directions in parentheses): C/1999 F2 - PA 230 (PA 98 $)$; C/1999 J2 - PA $18^{\circ}\left(\mathrm{PA} 80^{\circ}\right)$; C/1999 N4 $\mathrm{PA} 97^{\circ}\left(\mathrm{PA} 111^{\circ}\right) ; \mathrm{C} / 1999 \mathrm{~T} 2-\mathrm{PA} 150^{\circ}\left(\mathrm{PA} 220^{\circ}\right)$; $\mathrm{C} / 2000 \mathrm{H} 1$ - $\mathrm{PA} 90^{\circ}\left(\mathrm{PA} 76^{\circ}\right) ; \mathrm{C} / 2000 \mathrm{~K} 1$ - PA $150^{\circ}$ (PA $102^{\circ}$ ). One can find large difference in $\mathrm{C} / 1999 \mathrm{~J} 2$ and C/1999 T2 and a smaller one in C/2000 K1, implying a significant amount of tail curvature in these comets. This is supported for C/1999 J2 by the antitail observations by Fukushima et al. (2000) made two months before our observing run. In the meantime the Earth crossed the orbital plane and the tail turned significantly. A colour index of C/1999 J2 also supports a dusty tail (see the photometric analysis) where one may observe apparently long tails if the curvature affects the projection significantly. Neglecting this curvature we calculated the true lengths taking into account the projection effect. The results are $19 \times 10^{5} \mathrm{~km}(\mathrm{C} / 1999 \mathrm{~F} 2), 8.5 \times 10^{5} \mathrm{~km}$ $(\mathrm{C} / 1999 \mathrm{~J} 2), 3.2 \times 10^{5} \mathrm{~km}(\mathrm{C} / 1999 \mathrm{~N} 4), 1.2 \times 10^{5} \mathrm{~km}$ 

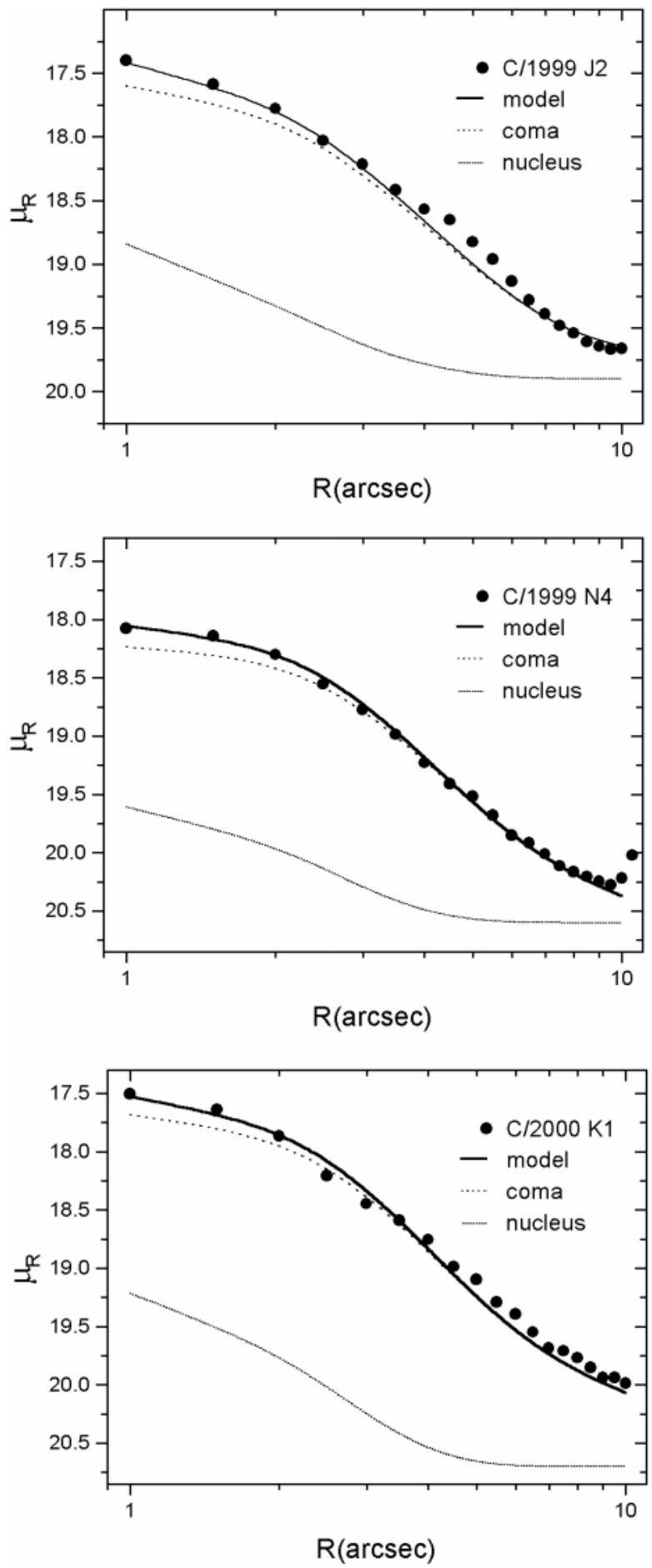

Fig. 2. $R$-filtered surface brightness distributions (in $\left.\mathrm{mag} / \operatorname{arcsec}^{2}\right)$ compared with the coma models. Note the logarithmic scale of the horizontal axes.

(C/1999 T2), $0.5 \times 10^{5} \mathrm{~km}(\mathrm{C} / 2000 \mathrm{H} 1)$ and $12 \times 10^{5} \mathrm{~km}$ (C/2000 K1).

Based on the test images taken previously on nonphotometric nights, three of the potential targets were excluded from further observations. The potential targets C/1999 T2 and C/2000 H1 were not really "distant" object during the observations. Furthermore, C/2000 H1 was quite compact and faint $(R=19)^{\mathrm{m}} 7$, integrated in the in-
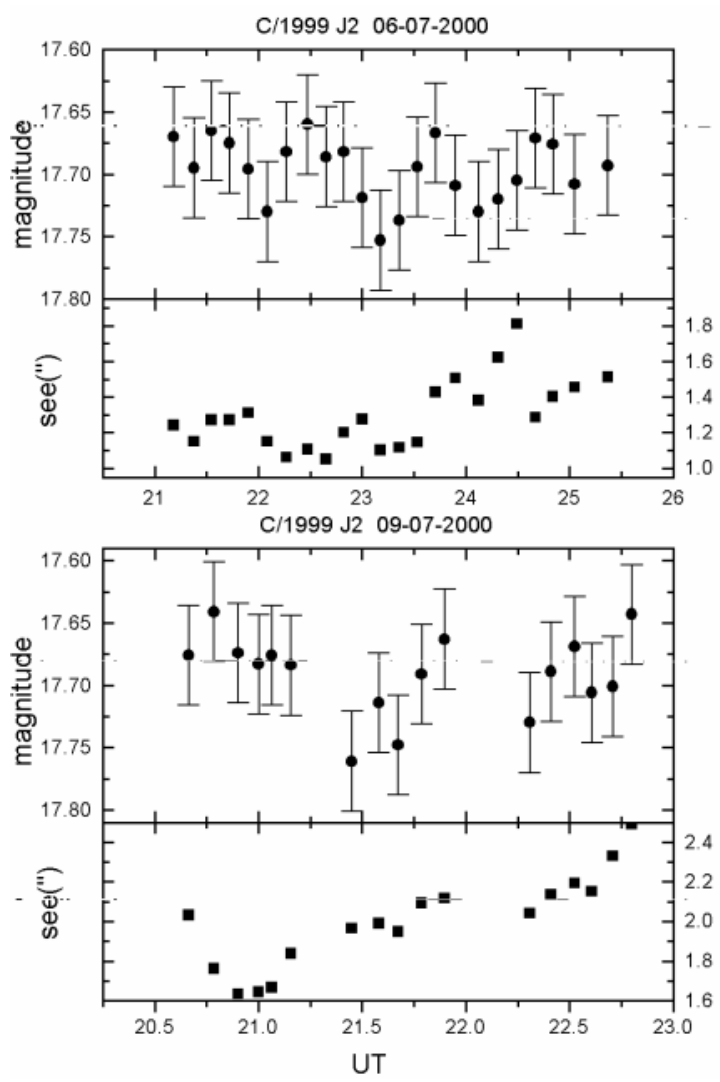

Fig. 3. The $R$ lightcurves of C/1999 J2 (Skiff) on July 6 and July 9, 2000. The lower panels show the seeing variations.

nermost $\left.6^{\prime \prime}\right) . \mathrm{C} / 1999 \mathrm{~T} 2$ was bright $\left(R=15^{\mathrm{m}} 9\right)$ with a strongly visible coma and $50^{\prime \prime}$-long tail. The integrated $R$ magnitude of $\mathrm{C} / 1999 \mathrm{~F} 2$ was $19{ }^{\mathrm{m}} 1$ in the inner $6^{\prime \prime}$, which is fainter than our practical limit. We note the impressive cyrrus-like tail as long as $350^{\prime \prime}$.

The surface brightness profiles (e.g. Jewitt \& Meech 1987; Lowry et al. 1999) were also calculated to examine the coma regularity. In spherically homogeneous and isotropic cases, the surface brightness $(B)$ profile bears a simple linear relation with a logarithmic derivative of -1 . Interaction between overstreaming matter and the radiation pressure modifies this value down to about -2 , according to the models by Jewitt \& Meech (1987). For our images the profiles were calculated by a model coma along with the nuclear brightness estimation.

The model comae were characterized by two free parameters, namely the slope parameter (logarithmic derivative of the surface brightness) and coma-to-nucleus brightness ratio. The resultant structure is a power-function of the radius with negative exponent, while the nucleus is represented with a delta-function in the center. To simulate the apparent motion and the effect of seeing, the model coma was convolved by the motion and the PSF (Lamy et al. 1998). The latter was determined from individual stellar profiles and its form was a simple Gaussian. We have performed a least-squares analysis, where the finally adopted parameters resulted in an appropriate fit of the observed surface brightness distribution. 
C/1999 J2 (Skiff)

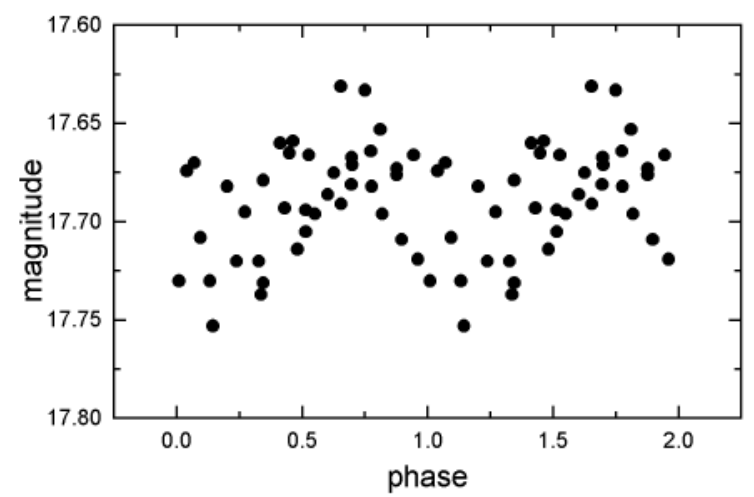

Fig. 4. The phase diagram of $\mathrm{C} / 1999 \mathrm{~J} 2$ (Skiff) $(P=1 \mathrm{~h})$.

The adopted numerical fits of the individual comae are examined in Fig. 2. A comparison of the measurements, the modelled coma components and their sums are presented. The measured brightnesses were averaged in neighbouring rings with a width of $0 . \prime 5$. As the background has been taken into account as an additive constant, the effect has been taken into account in this representation too. This allows us to show the photometric data with respect to the real limit of the sky conditions.

Determination of the absolute brightness of the coma allows one to calculate the apparent magnitude of the core, which results in an estimate of the diameter of the solid nucleus. Certainly, as the coma strongly affects the brightness of the nucleus, the apparent brightness of the nucleus can be determined with quite large errors. In the case of the present calculations, the coma contamination was about 1.5-2 magnitudes, therefore, the confidence interval of the estimated diameters may be in the range of half to twice the accepted values.

From the $R$-band nuclear magnitudes the mean optical cross sections of the nucleii were determined using the equation (Eddington 1910)

$p_{\mathrm{R}} \bar{C}=\frac{2.25 \times 10^{22} \pi R^{2} \Delta^{2} 10^{0.4\left(\mathrm{~m}_{\mathrm{Sun}}-\overline{\mathrm{mR}}\right)}}{10^{-0.4 \alpha \beta}}$

where $p_{R}$ is the red geometric albedo, $\bar{C}$ is the mean cross section, $m_{\text {Sun }}=-27.96 \mathrm{mag}$ is the $R$-band magnitude of the Sun and $\overline{m_{R}}$ is the mean $R$ magnitude of the comet, while $\beta$ is usually assumed to be 0.04 (Luu \& Jewitt 1992). An important question is the involved sytematic error, which is very difficult to estimate. The brightness profile of the coma may be distorted by anisotropic substructures (e.g. jets, bright patches) blurred by the seeing. Therefore one can barely calculate the brightness of the nuclei in the case of ground-based observations, and the resulted nuclear diameters are often overestimated (I. Tóth, personal communication).

The remarks on the individual comets are as follows.

C/1999 J2 (Skiff)

The object was detected for the first time on CCD frames of the LONEOS-Schmidt $(59 \mathrm{~cm})$ telescope on May 13,
1999 for the first time (Skiff et al. 1999). This comet with a discovery brightness of $16^{\mathrm{m}} 0$ has the third largest perihelion distance known (7.110 AU, the transit was on April 5, 2000). The apparent total visual brightness was 14.5 during the observations, and because of its high declination it was a favourable target for observers in the northern hemisphere. The derived absolute brightness is 3.0 , which is quite bright compared to other similar comets. The intrinsic peculiarity of this comet was also suggested by the dust antitail reported by Fukushima et al. (2000) in May, 2000 .

The observed $V-R$ colour index of the inner coma is $0.25 \pm 0.05$. A weak ion tail is barely visible on the direct images, and therefore the relatively bluish colour may be attributed to possible $C_{2}$ emission in the $V$-band. The obtained coma-corrected absolute photometry gave the following mean nuclear brightness: $\langle R\rangle=19{ }^{\mathrm{m}} 9 \pm 1^{\mathrm{m}} .0$. The coma contamination was estimated to be $87 \pm 6 \%$ of the total inner intensity (formal error). Assuming 0.04 albedo, the calculated cross section is $p_{R} C=4 \pm 3 \mathrm{~km}^{2}$ resulting in a nuclear diameter of $10 \pm 8 \mathrm{~km}$. This is a quite large value, however, it is simply necessary to support the tremendous activity observed. The logarithmic coma brightness density is linear in the inner $13^{\prime \prime} .0$ with a slope of $-1.6 \pm 0.1$. This value is significantly larger than the expected one for an isotropic steady-state coma and suggests strong interaction of the outflow and the radiation pressure.

Time-series observations showed that there were rapid small-amplitude variations on a time-scale of an hour, though with small significance. We present the lightcurves in Fig. 3 (the data regard to $1^{\prime \prime} 3$ aperture radius). In order to quantify the cyclic variation, a standard Fourieranalysis was performed. A very short period of $0.96 \pm 0.07$ was revealed, the phase diagram is plotted in Fig. 4.

As rotation is the easiest way to explain light variability, we have compared the observed behaviour with the rotational breakup calculations of Davidsson (1999). Accepting rotational variability, the period of rotation is twice the period of the lightcurves. This means approximately 2 hours for the rotational period, which is physically permitted for thopse bodies which are smaller than $4 \mathrm{~km}$ in spherical approximation. This does not contradict the estimated diameter of the nucleus. However, other alternative mechanisms cannot be exluded, which are presently unknown. It is worthwhile noting that a similarly fast oscillation has been found for the asteroid 1689 Floris-Jan (Pych 1999), which has long rotational period. The rapid variations were suggested to be caused by secondary rotational effects, though no firm physical explanation was drawn.

\section{$C / 1999 N_{4}($ LINEAR)}

This retrograde comet was found as an asteroid by the LINEAR project on July 12, 1999 and its unusual motion raised the question of its real nature (Tichy et al. 1999). The discovery was made almost a year before the perihelion at 5.505 $\mathrm{AU}$, therefore, the evolution of this object 

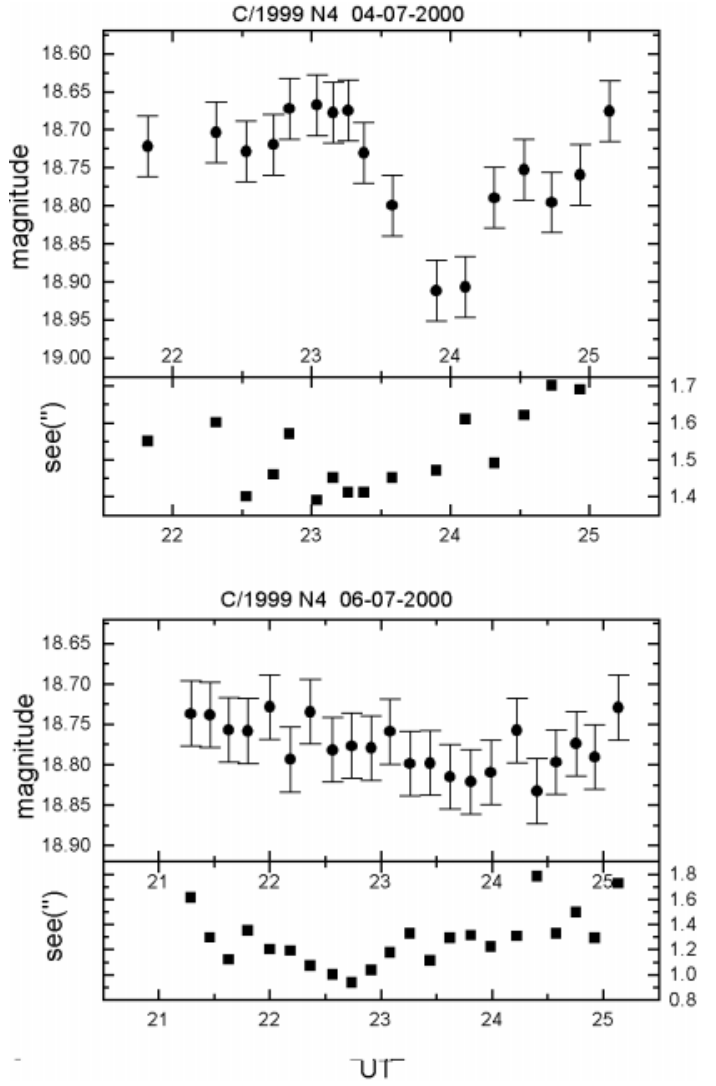

Fig. 5. The lightcurve of C/1999 N4 (LINEAR) on July 4 and July 6, 2000.

could be well monitored. The initial brightness of $17^{\mathrm{m}} .5$ brightened up to $15{ }^{\mathrm{m}} 0$.

Our measurements were taken on 2 nights. On July $4 / 5$ the comet showed a light variation of $0 .{ }^{\mathrm{m}} 3$, the most striking feature of which is the rapid dimming between 23.2 - 01 UT. The lightcurve is presented in Fig. 5. On the second night (July 6) we could detect only an ambiguous variation with an amplitude not exceeding 0.08 , while a rotation effect would have been expected on this night too. To exclude the correlation with the seeing, the seeing variation is also presented below the lightcurves.

The $V-R$ colour index of the inner coma is $0.47 \pm 0 .{ }^{\mathrm{m}} 05$, fairly close to the solar value $\left((V-R)_{\odot}=\right.$ 0. 36 , Meech et al. 1995). This implies a relatively simple reflection with no emission and little dust. The obtained coma-corrected absolute photometry gave the following mean nuclear brightness: $\langle R\rangle=20{ }^{\mathrm{m}} 6 \pm 0 \mathrm{~m} 2$. The coma contamination was estimated to be $90 \pm 5 \%$ of the total inner intensity (formal error). Assuming 0.04 albedo, the calculated cross section is $p_{R} C=0.4 \pm 0.3 \mathrm{~km}^{2}$ resulting in a nuclear diameter of $3 \pm 2 \mathrm{~km}$. The logarithmic surface brightness profile is the same as for the coma of C/1999 J2: a linear relation in the inner 8 .! 0 with a slope of $-1.7 \pm 0.1$.

\section{$C / 2000 K 1$}

The retrograde comet was discovered at a brightness of 18 . 0 by the LINEAR project in May 2000 (Shelly et al. 2000). Further prediscovery images were found on frames
C/2000 K1 04-06-2000

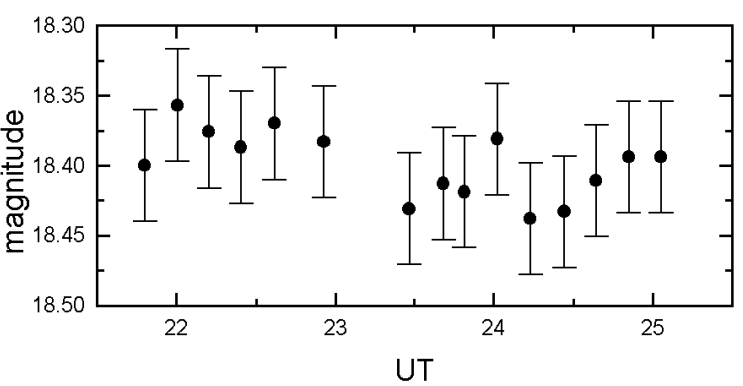

Fig. 6. The lightcurve of $\mathrm{C} / 2000 \mathrm{~K} 1$ (LINEAR) on July 4, 2000 .

from the previous year (Amburgey \& Zoltowski 2000). The perihelion passage occurred at 6.276 AU and a visual brightness of 14.5 .

We have found no significant variation during the observing run (Fig. 6). Unfortunately, we could obtain only the presented 3-hour data series which does not allow any firm conclusion to be drawn. The $V-R$ colour index of the inner coma is $0{ }^{\mathrm{m}} 68 \pm 0{ }^{\mathrm{m}} 05$, slightly reddish. This means a considerably dusty coma. The obtained comacorrected absolute photometry gave the following mean nuclear brightness: $\langle R\rangle=19 \mathrm{~m} 5 \pm 1 \mathrm{~m} 0$. The coma contamination was estimated to be $84 \pm 10 \%$ of the total inner intensity (formal error). Assuming 0.04 albedo, the calculated cross section is $p_{R} C=5 \pm 3 \mathrm{~km}^{2}$ resulting in a nuclear diameter of $11 \pm 8 \mathrm{~km}$. As for C/1999 J2, the remarkable tail and coma activity requires a large nucleus. The recorded logarithmic surface brightness profile has a slope of $-1.55 \pm 0.1$ determined in the inner $14^{\prime \prime} .0$.

\section{Discussion}

The presented observations revealed some basic parameters of three interesting distant comets. Despite the large heliocentric distances of 5.5-7.2 AU, we could observe extended tails and fairly bright comae. The canonical view of cometary activity at such distances excludes the possibility of water ice sublimation as the inner "engine" of the gas and dust production, because the heat from the Sun is not sufficient. Usually, it is assumed that a COor $\mathrm{CO}_{2}$-dominated nucleus may be the source of this activity (Houpis \& Mendis 1981; Luu 1993). Bar-Nun \& Prialnik (1988) discussed the possible hydrogen comae in distant comets as a further mechanism beyond $\sim 4 \mathrm{AU}$. Based on a very extensive database of comet observations ( $\sim 50$ comets over a range of 1 to $\sim 30$ AU), Meech \& Hainaut (1997) have clearly shown that dynamically young comets are intrinsically brighter, exhibiting dust comae and activity at large distances in the region where water ice sublimation is not possible. Our observations fit very well the description above.

Because of the unexpectedly strong coma contamination, the presented results may suffer from large systematic errors, as the applied coma subtraction is likely an oversimplification of the real case. Thus, the calculated 
nuclear diameters should be considered as well-educated guesses with possibly large systematic uncertainties (up to $5-10 \mathrm{~km}$ ). The recorded surface brightness profiles with logarithmic derivatives $-1.6 \ldots-1.8$ are characteristic for isotropic steady-state outflows affected by the radiation pressure alone (Jewitt 1991; Luu 1993).

Finally, the main observational results can be summarized as follows:

1. CCD observation of three distant comets taken on three night in July, 2000 are presented. The time-series data revealed short-term variations in two comets, while the third one was constant at a level of \pm 0 m 05 . As the time span is quite short, no firm conclusion was drawn on the possible rotation.

2. The absolute photometry was corrected for the presence of comae and nuclear radii were determined assuming a 0.04 albedo. The results are: C/1999 J2 - $10 \mathrm{~km}, \mathrm{C} / 1999$ $\mathrm{N} 4-3 \mathrm{~km}, \mathrm{C} / 2000 \mathrm{~K} 1-11 \mathrm{~km}$.

3. The observed $V-R$ colours imply a slightly reddish coma for $\mathrm{C} / 2000 \mathrm{~K} 1$, a normal solar reflected light for C/1999 N4 and a slightly bluish coma for C/1999 J2. The latter one may be caused by the $C_{2}$ emission decreasing the $V$-magnitude. The ion tail of $\mathrm{C} / 1999 \mathrm{~J} 2$ was also detected.

4. We note that our results are strongly affected by selection effects, as the target list was formed to include the brightest and most distant active comets visible in July, 2000.

5. As a by-product of this project, coma and tail characteristics of three other comets have been also examined. The results are also discussed, although two of these targets were not further than $4 \mathrm{AU}$.

Acknowledgements. This research was supported by Pro Renovanda Cultura Hungariae Grants DT 2000. máj./43., DT 2000. máj./44., DT 2000. máj./48. and DT 1999. ápr./23, FKFP Grant 0010/2001, OTKA Grant \#T032258, "Bolyai János" Research Scholarship of LLK from the Hungarian Academy of Sciences and Szeged Observatory Foundation. The referee, Dr. Olivier Hainaut, helped to improve the paper with his comments and suggestions. The warm hospitality and helpful assistance of the staff of Calar Alto Observatory is gratefully acknowledged. The NASA ADS Abstract Service was used to access data and references.

\section{References}

Amburgey, L., \& Zoltowski, F. B. 2000, IAUC, No. 7431, 2 Boehnhardt, H., Rainer, N., Birkle, K., \& Schwehm, G. 1999, A\&A, 341, 912
Davidsson, B. J. R. 1999, Icarus, 142, 525.

Eddington, A. S. 1910, MNRAS, 70, 442

Fay, T., \& Wisniewsky, W. 1978, Icarus, 34, 1

Fukushima, T., Nakajima, T., \& Watanabe, J. 2000, IAUC, No. 7415,3

Hainaut, O. R., \& Meech, K. J. 1997, Interaction between Planets and Small Bodies, 23rd meeting of the IAU, Joint Discussion 6, Kyoto, Japan

Houpis, H. L. F., \& Mendis, D. A. 1981, M\&P, 25, 397

Jewitt, D. 1990, AJ, 351, 277

Jewitt, D. 1991, in Comets in the Post-Halley Era, ed. R. Newburn, Jr., M. Neugebauer, \& J. Rahe (Dordrecht, Kluwer Acedemic), 19

Jewitt, D. 1992, in Observations and Physical Properties of Small Solar System Bodies, ed. A. Brahic, J. Gerard, \& J. Surdej, Liège: Inst. Astrophys., 85

Jewitt, D., \& Meech, K. 1985, Icarus, 64, 329

Jewitt, D., \& Meech, K. 1987, ApJ, 317, 992

Jorda, L., Lamy, P. L., \& Groussin, O., et al. 2000, ESA-SP 455, 61

Lamy, P. L., \& Tóth, I. 1995, A\&A, 293, L43

Lamy, P. L., Tóth, I., \& Weaver, H. A. 1998, A\&A, 337, 945

Lamy, P. L., Tóth, I., A'Hearn, M. F., et al. 1999, Icarus, 140, 424

Landolt, A. U. 1992, AJ, 104, 1,

Licandro, J., Serra-Ricart, M., Oscoz, A., et al. 2000a, AJ, 119, 3133

Licandro, J., Tancredi, G., Lindgren, M., et al. 2000, Icarus, 901, 161

Lowry, S. C., Fitzsimmons, A., Cartwright, I. M., \& Williams, I. P. 1999, A\&A, 349, 649

Luu, J. X. 1993, PASP, 105, 946

Luu, J. X., \& Jewitt, D. C. 1992, AJ, 104, 2243

Meech, K. J. 1991, in Comets in the post-Halley era, vol. 1, 629

Meech, K. J., Belton, M., Mueller, B., et al. 1993, AJ, 106, 1222

Meech, K. J., Knopp, G. P., \& Farnham, T. L. 1995, Icarus, 116,46

Meech, K. J., Bauer, J., \& Hainaut, O. 1997, A\&A, 326, 1268

Meech, K. J., \& Hainaut, O. R. 1997,

O'Ceallaigh, D. P., Fitzsimmons, A., \& Williams, I. P. 1995, A\&A, 297, L17

Pych, W. 1999, A\&A, 343, L75

Shelly, F., Blythe, M., Bezpalko, M., et al. 2000, IAUC, No. 7430,1

Skiff, B. A., Koehn, B. W., Tichy, M., \& Moravec, Z. 1999, IAUC, No. 7165, 1

Tichy, M., Sarounova, L., \& Zoltowski, F. B. 1999, IAUC, No. 7226,1 